\title{
El rol de la nutrición en los cuidados paliativos
}

\author{
The role of nutrition in palliative care \\ O papel da nutrição nos cuidados paliativos
}

\author{
Evelyn Frías-Toral, MD, MSc*
}

https://doi.org/10.35454/rncm.v4n2.275

El colaborar por más de una década en el hospital oncológico de mayor referencia nacional, la Sociedad de Lucha contra el Cáncer del Ecuador (SOLCA), me permitió valorar mucho el impacto positivo de dar los cuidados paliativos (CP) oportunos y adecuados a estos pacientes. Esta institución fue la primera a nivel nacional en satisfacer la demanda creciente de estos servicios especializados. El manejo integral de los aspectos nutricionales incide positivamente tanto en la evolución de la enfermedad como en la calidad de vida de estos enfermos. Es por ello que consideré necesario proponer a la distinguida editora de la Revista de Nutrición Clínica y Metabolismo, órgano de difusión científica de gran prestigio, este número temático, para presentar el trabajo armonioso de estas dos especialidades en el manejo de pacientes con enfermedades incurables, en estadio avanzados, fuera de recursos terapéuticos, y terminales.

De acuerdo con el consenso de la International Association for Hospice \& Palliative Care (IAHPC), los CP se redefinieron en el 2020 como "El cuidado holístico activo de personas de todas las edades con un sufrimiento grave relacionado con la salud (por sus siglas en inglés, SHS) debido a una enfermedad grave, y especialmente de aquellos que se acercan al final de la vida. Tiene como objetivo mejorar la calidad de vida de los pacientes, sus familias y sus cuidadores" ${ }^{\prime 1)}$.

La evaluación e intervención nutricional constituyen algunos de los pilares fundamentales en los pacientes que se benefician de los CP, puesto que directamente recaen

*Correspondencia: Evelyn Frías Toral. evelyn.frias@cu.ucsg.edu.ec en el dominio, identificado por la National Coalition for Hospice and Palliative Care-National Consensus Project, relacionado con el manejo de síntomas, entre los cuales están la pérdida de peso, la debilidad y la fatiga ${ }^{(2)}$. Adicionalmente, el crecimiento exponencial de pacientes pediátricos y adultos con requerimientos de $\mathrm{CP}$, por presentar algún SHS engloba una variedad de enfermedades y condiciones, como anomalías congénitas, condiciones neonatales, insuficiencia cardíaca, enfermedades neurológicas progresivas, enfermedad renal crónica, enfermedad hepática avanzada, enfermedad pulmonar obstructiva crónica, SIDA, diabetes, cáncer, entre otras ${ }^{(3,4)}$.

A nivel mundial se estima que 40 millones de personas precisan de CP, de los cuales el $50 \%$ se encuentra en etapa terminal. Es destacable que al fomentar la implementación temprana de $\mathrm{CP}$, en conjunto con intervenciones nutricionales precisas en estas enfermedades, se puede reducir el número de admisiones hospitalarias y mejorar la calidad de vida de estos pacientes ${ }^{(5-7)}$. Todo lo cual debe ser analizado a detalle por un equipo multidisciplinario, que estime los riesgos y beneficios para el requerimiento individual de estos enfermos. Además, dichas recomendaciones deben estar alineadas a los principios éticos y al respeto de los deseos del paciente y familia en relación con el manejo terapéutico de éste ${ }^{(8)}$.

Es importante resaltar que los requerimientos nutricionales especiales de este grupo particular de pacientes son cubiertos por varios principios de la Declaración de Cartagena (DC), cuyo objetivo principal es promover el cuidado nutricional y la lucha contra la malnutrición ${ }^{(9)}$. Esta última resulta muy común en estos pacientes, que algunas veces llegan a su desenlace anticipadamente por alguna complicación relacionada con su estado o 
manejo nutricional antes que por su afección de base. Resulta fundamental alimentar con dignidad al paciente de $\mathrm{CP}$, con recomendaciones integrales que respeten la autonomía del paciente (DC principio 1,5). El cuidado nutricional implica un proceso basado en un enfoque multidisciplinario, que considere las preferencias del paciente, y que debe darse de forma sistematizada en todos los usuarios de CP (DC principios 2-4).

Como lo presentan varios autores de los artículos de este número especial, existe una necesidad de más evidencia científica que promueva la implementación de guías nutricionales en los pacientes de $\mathrm{CP}$ a nivel regional e internacional, lo cual conlleva un trabajo en conjunto de los profesionales implicados en el manejo de estos pacientes (DC principios 7, 9 y 13 ).

Por todo lo antes mencionado, en este número temático, líderes de opinión regionales e internacionales, representantes de las diferentes especialidades involucradas en los CP (Nutrición Clínica, Bioética, Medicina Interna, Cirugía, Oncología, Pediatría, Psicología, Enfermería, entre otros) presentarán información actualizada basada en la evidencia y en la experiencia acumulada a lo largo de su práctica atendiendo las necesidades específicas de este grupo especialmente vulnerable de pacientes. De esta forma, el objetivo de este número es difundir la importancia de la adopción temprana de los CP, en conjunto con la evaluación y tratamiento nutricional oportuno para el manejo integral de los pacientes con SHS.

Debido el requerimiento ascendente de los CP a nivel mundial, el primer artículo de este número especial expone los conceptos básicos de los CP. Santacruz y colaboradores presentan la definición de los CP, su desarrollo histórico y el papel de la nutrición en $\mathrm{CP}^{(10)}$. La Presidente de la Asociación Latinoamericana de CP, Patricia Bonilla, describe el progreso de los CP en Latinoamérica en relación con los siguientes dominios: educación, disponibilidad de opioides, investigación, políticas públicas e implementación de servicios ${ }^{(11)}$. La docente, investigadora y editora de la revista, Diana Cárdenas, en su revisión responde dos preguntas fundamentales en relación con los CP: ¿cuándo se deben iniciar y detener la terapia nutricional? y ila terapia nutricional mejora la calidad de vida y prolonga la sobrevida de los pacientes en $\mathrm{CP}$ ? $^{(12)}$.

Los CP tienen un profundo impacto en la autonomía del paciente, es por ello por lo que el profesor Barrocas y colaboradores exponen a detalle las perspectivas éticas de los CP y la nutrición. Describen el abordaje norteamericano para lograr intervenciones exitosas en $\mathrm{CP}$, con base en el respeto, la comunicación y el trabajo en equipo (por sus siglas en inglés: Accountability, Communication and Teamwork - ACT) y delinean la relación de la Declaración de Cartagena en los CP para alcanzar una atención centrada en el paciente. Finalmente aterrizan esos conceptos en el manejo específico de pacientes con demencia avanzada ${ }^{(13)}$.

La académica de carrera temprana de ESPEN, Muscogiuri y colaboradores, resumen las recomendaciones generales y específicas de ESPEN para el manejo nutricional de estadios avanzados de las principales enfermedades que se benefician de $\mathrm{CP}^{(14)}$. Vallejo $\mathrm{y}$ colaboradores especifican las pautas de la alimentación oral, como componente del esquema de CP, en pacientes con cáncer en etapa terminal de la enfermedad ${ }^{(15)}$. Sabogal y colaboradores nos comparten la experiencia adquirida en el tratamiento nutricional de pacientes oncológicos en estadios avanzados, o con pobre pronóstico del Servicio de Cirugía Hepatobiliar y Pancreática del Hospital Méderi en Colombia. Explican el abordaje empleado, el cual se basa en un enfoque humano, con especial atención en el fortalecimiento de la relación médico-paciente ${ }^{(16)}$.

El profesor brasileño Zorzo y colaboradores analizan las indicaciones y contraindicaciones de la hidratación y nutrición artificial en una población aún más vulnerable: la pediátrica en CP. Resaltan la importancia de una evaluación minuciosa y continua para determinar las mejores opciones de tratamiento nutricional en estos pacientes. En esta toma de decisiones se destaca la relevancia de una participación conjunta del equipo de atención multidisciplinar, el paciente y su familia para alcanzar dicho objetivo $^{(17)}$. Los líderes uruguayos del proyecto Extension for Community Healthcare Outcomes (ECHO) para Latinoamérica en CP profundizan la trascendencia del manejo nutricional del paciente, el abordaje completo y la repercusión en la esfera emocional no solo del enfermo, sino del núcleo familiar ${ }^{(18)}$. Para concluir, de forma práctica Maza y colaboradores detallan el manejo de un caso avanzado de adenocarcinoma gástrico con nutrición parenteral intrahospitalaria; además, exponen las limitaciones existentes en la región, de contar con nutrición parenteral en casa a favor de estos pacientes ${ }^{(19)}$.

Por último, pero no menos importante, deseo expresar mis agradecimientos especiales a las profesoras Dolores Rodríguez (Nutrición Clínica) y Mariana Vallejo (Cuidados Paliativos) por ser excelentes mentoras y haberme motivado a profundizar mi práctica profesional y de investigación en esta población con requerimientos especiales. Es mi deseo sincero el que este número temático sirva para promover las recomendaciones nutri- 
cionales en los cuidados paliativos y para sentar las bases para futuras investigaciones en este ámbito.

\section{Conflicto de intereses}

La autora declara no tener conflicto de interés.

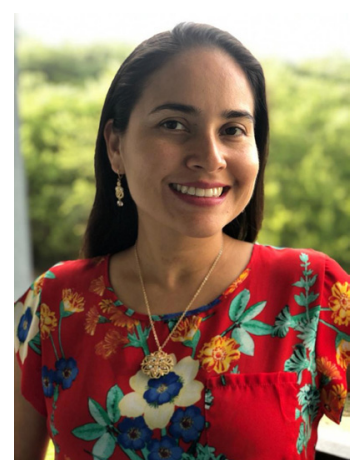

Evelyn Frias-Toral, MD, MSc. Médico y Cirujano General de la Universidad Católica de Santiago de Guayaquil. Magíster en Investigación Clínica y Epidemiológica de la Universidad de Guayaquil con un Diplomado en Nutrición Enteral y Parenteral Aplicada de la Universidad de Montrer, México. Es Profesora Asociada de Metodología de Investigación del Posgrado de Cuidados Paliativos de la Universidad Católica Santiago de Guayaquil. Forma parte del Comité Científico, de Enseñanza y Publicaciones de ASENPE (Asociación Ecuatoriana de Nutrición Parenteral y Enteral) y es su representante ante el Consejo Internacional de ESPEN (European Society of Clinical Nutrition and Metabolism). Ha obtenido varias distinciones entre ellas ser parte del primer grupo de ESPEN Early Career Faculty lo cual le ha permitido participar activamente en varios proyectos de ESPEN, como nutritionDay, entre otros. Se desempeña como Editora Asociada de las revistas Clinical Nutrition ESPEN y Revista de Nutrición Clínica y Metabolismo.

\section{Referencias bibliográficas}

1. Radbruch L, De Lima L, Knaul F, Wenk R, Ali Z, Bhatnaghar $\mathrm{S}$, et al. Redefining palliative care- A new consensus-based definition. J Pain Symptom Manage. 2020;60(4):754-64. doi: 10.1016/j.jpainsymman.2020.04.027.

2. National Coalition for Hospice and Palliative Care. Clinical practice guidelines for quality palliative care. 4 . $^{\text {a }}$ edición. 2018. Disponible en: https://www.nationalcoalitionhpc.org/ncp

3. Connor SR, Downing J, Marston J. Estimating the global need for palliative care for children: a cross-sectional analysis. J Pain Symptom Manage. 2017;53(2):171-7. doi: 10.1016/j.jpainsymman.2016.08.020.

4. Knaul FM, Farmer PE, Krakauer EL, De Lima L, Bhadelia A, Jiang KweteX, et al. Alleviating the access abyss in palliative care and pain relief-an imperative of universal health coverage: the Lancet Commission report. Lancet. 2018;391(10128):1391454. doi: 10.1016/S0140-6736(17)32513-8.

5. Global atlas of palliative care at the end of life [Internet]. Worldwide Hospice Palliative Care Alliance and World Health Organization. [Consultado el 30 de octubre de 2020]. Disponible en: http://www.thewhpca.org/resources/global- atlas-on-end-of-life-care?task=callelement \&;format=raw\&i tem_id=93\&element $=f 85 c 494 b-2 b 32-4109-b 8 c 1-083 c c a 2 b$ $7 \mathrm{db} 6 \&$ method $=$ download\&args $[0]=941140 \mathrm{f} 7 \mathrm{c} 369715 \mathrm{aebc}$ 1c2bbdacelee3

6. Bozzetti F. Is there a place for nutrition in palliative care? Support Care Cancer. 2020;28(9):4069-75. doi: 10.1007/ s00520-020-05505-x.

7. McGinley E. Role of nutrition in the final stages of palliative care. J Community Nurs. 2015;29(1):53.

8. Druml C, Ballmer PE, Druml W, Oehmichen F, Shenkin A, Singer $\mathrm{P}$, et al. ESPEN guideline on ethical aspects of artificial nutrition and hydration. Clin Nutr. 2016;35(3):545-56. doi: 10.1016/j.clnu.2016.02.006.

9. Cárdenas D, Bermúdez CE, Echeverri S, Pérez A, Puentes M, López L, et al. Declaración de Cartagena. Declaración Internacional sobre el Derecho al Cuidado Nutricional y la Lucha contra la Malnutrición. Nutr Hosp. 2019;36(4):97480. doi: 10.20960/nh.02701.

10. Santacruz Escudero JG, Martínez Gil LF. Cuidados paliativos: conceptos básicos. Rev Nutr Clin Metab. 2021;4(2):14-18. https://doi.org/10.35454/rncm.v4n2.212.

11. Bonilla P. Cuidados paliativos en Latinoamérica. Rev Nutr Clin Metab. 2021;4(2):4-13. https://doi.org/10.35454/ rncm.v4n2.226.

12. Cárdenas D. ¿Cómo alimentar al paciente en cuidados paliativos? Una revisión narrativa. Rev Nutr Clin Metab. 2021;4(2):50-58. https://doi.org/10.35454/rncm.v4n2.243.

13. Barrocas C, Schwartz DB, Echeverri S. Perspectiva ética en la nutrición y los cuidados paliativos: un enfoque en la atención a los pacientes con demencia avanzada. Rev Nutr Clin Metab. 2021;4(2):24-42. https://doi.org/10.35454/rncm.v4n2.244.

14. Muscoguiri G, Barrea L, Carignano MA, Ceriani F. Recomendaciones nutricionales en cuidados paliativos para pacientes con enfermedades avanzadas y al final de la vida. Rev Nutr Clin Metab. 2021;4(2):59-70. https://doi. org/10.35454/rncm.v4n2.272.

15. Vallejo Martínez M, Baque Hidalgo JE. Alimentación y cuidados paliativos en el paciente oncológico terminal. Rev Nutr Clin Metab. 2021;4(2):71-77. https://doi.org/10.35454/ rncm.v4n2.274.

16. Sabogal JC, Santos García C, Ortiz AC, Rey Chávez CE, Conde Monroy D. Nutrición al final de la vida: perspectiva paliativa del cirujano. Rev Nutr Clin Metab. 2021;4(2):78-85. https://doi.org/10.35454/rncm.v4n2.220.

17. Zorzo RA, de Arruda Leite F, Cardeal da Costa Zorzo J. Nutrición en el paciente pediátrico en cuidados paliativos. Rev Nutr Clin Metab. 2021;4(2):43-49. https://doi. org/10.35454/rncm.v4n2.218.

18. Ramos L, Loureiro V, Piriz Álvarez G. Aspectos psicológicos de la nutrición en cuidados paliativos. Rev Nutr Clin Metab. 2021;4(2):19-23. https://doi.org/10.35454/rncm.v4n2.260.

19. Maza C. Abordaje nutricional de un paciente en cuidados paliativos. Análisis de un caso clínico. Rev Nutr Clin Metab. 2021;4(2):86-90. https://doi.org/10.35454/rncm.v4n2.233. 